University of South Carolina

Scholar Commons

$3-2011$

\title{
Validation of the Actical Activity Monitor in Middle-Aged and Older Adults
}

Steven P. Hooker

University of South Carolina - Columbia

Anna Feeney

Brent E. Hutto

Karin A. Pfeiffer

Kerry Mclver

See next page for additional authors

Follow this and additional works at: https://scholarcommons.sc.edu/

sph_epidemiology_biostatistics_facpub

Part of the Public Health Commons

\section{Publication Info}

Published in Journal of Physical Activity and Health, Volume 8, Issue 3, 2011, pages 372-381.

Hooker, S. P., Feeney, A., Hutto, B., Pfeiffer, K. A., Mclver, K., Heil, D. P., ... Blair, S. N. (2011). Validation of the actical activity monitor in middle-aged and older adults. Journal of Physical Activity and Health, 8(3), 372-381.

(c) Journal of Physical Activity and Health, 2011, Human Kinetics

This Article is brought to you by the Epidemiology and Biostatistics at Scholar Commons. It has been accepted for inclusion in Faculty Publications by an authorized administrator of Scholar Commons. For more information, please contact digres@mailbox.sc.edu. 


\section{Author(s)}

Steven P. Hooker, Anna Feeney, Brent E. Hutto, Karin A. Pfeiffer, Kerry Mclver, Daniel P. Heil, John E. Vena, Michael J. LaMonte, and Steven N. Blair 


\title{
Validation of the Actical Activity Monitor in Middle-Aged and Older Adults
}

\section{Steven P. Hooker, Anna Feeney, Brent Hutto, Karin A. Pfeiffer, Kerry Mclver, Daniel P. Heil, John E. Vena, Michael J. LaMonte, and Steven N. Blair}

\begin{abstract}
Purpose: This study was designed to validate the Actical activity monitor in middle-aged and older adults of varying body composition to develop accelerometer thresholds to distinguish between light and moderate intensity physical activity (PA). Methods: Nonobese 45 to $64 \mathrm{yr}(\mathrm{N}=29)$, obese 45 to $64 \mathrm{yr}(\mathrm{N}=21)$, and $\geq 65$ yr ( $\mathrm{N}=23$; varying body composition) participants completed laboratory-based sitting, household, and locomotive activities while wearing an Actical monitor and a portable metabolic measurement system. Nonlinear regression analysis was used to identify activity count (AC) cut-points to differentiate between light intensity (<3 METs) and moderate intensity ( $\geq 3 \mathrm{METs}$ ) PA. Results: Using group-specific algorithms, AC cut points for 3 METs were 1634, 1107, and 431 for the obese 45 to 64 yr group, nonobese 45 to 64 yr group, and $\geq 65$ yr group, respectively. However, sensitivity and specificity analysis revealed that an AC cut-point of 1065 yielded similar accuracy for detecting an activity as less than or greater than $3 \mathrm{METs}$, regardless of age and body composition. Conclusion: For the Actical activity monitor, an AC cut-point of 1065 can be used to determine light and moderate intensity PA in people $\geq 45$ years of age.
\end{abstract}

Keywords: physical activity, assessment, accelerometer, obesity

Accurate estimation of daily physical activity (PA) is important for epidemiological and experimental studies because low levels of PA are strongly associated with increased risk of cardiovascular disease, diabetes, cancer, and other chronic conditions. ${ }^{1}$ However, there remains a need for valid and reliable quantitative methods of assessing PA that are feasible for use in field settings, especially those involving large numbers of participants (ie, $>1000$ ).

Accelerometry has emerged as a popular means of monitoring PA patterns. Accelerometers are portable, unobtrusive devices that provide a direct and objective method of assessing free-living PA and reduce the random and systematic errors associated with the self-report measures commonly used in epidemiological studies. ${ }^{2}$ Activity monitors are also advantageous because of their small size and light weight, long-term data storage capabilities, acceptability to participants, and capacity to assess the intensity, frequency, and duration domains of PA. ${ }^{3}$

Hooker, Feeney, and Hutto are with the Prevention Research Center, University of South Carolina, Columbia, SC. Pfeiffer is with the Dept of Kinesiology, Michigan State University, Lansing, MI. McIver and Blair are with the Dept of Exercise Science, University of South Carolina, Columbia, SC. Heil is with the Dept of Health and Human Development, Montana State University, Bozeman, MT. Vena is with the Dept of Epidemiology and Biostatistics, University of South Carolina, Columbia, SC. LaMonte is with the Dept of Social \& Preventive Medicine, University at Buffalo, Buffalo, NY.
Despite apparent advantages, the interpretation of accelerometer data remains a challenge due to gaps and inconsistencies in the validation literature. 4 To date, reported cut-points for moderate and vigorous intensity PA (MVPA) for adults have varied considerably within and between brands of accelerometers. ${ }^{5,6}$ The vast majority of data have been derived from studies of healthy, younger adults (age 18 to 40 years) in the normal or slightly overweight range of body weights. Compared with other age groups, older adults spend a higher percentage of waking hours performing low intensity PA and a lower percentage doing vigorous intensity PA. ${ }^{7-11}$ Age- or weight-related changes in basal metabolic rate and fat free mass may also contribute to alterations in the energy expenditure associated with any given PA and related accelerometer activity count. ${ }^{12}$ Therefore, MVPA cut-points generated from a young, nonobese sample may lead to misclassification of PA levels and inaccurate prediction of activity energy expenditure (AEE) in persons who are older and/or obese. As a result, MVPA cut-points should be based on data from a sample that is representative of the population being studied and activities common to the age of the participants in the study, with locomotive activities (eg, walking) being of primary interest. ${ }^{13}$

Thus, to fill a major gap in the existing research, the purpose of this study was to validate the Actical accelerometer for use in middle-aged and older adults of varying body composition. In addition, the specific intentions of this study were to generate either group-specific 
cut-points or an overall standard cut-point that could accurately differentiate between light and moderateintensity PA which could be applied to large populations of such persons in longitudinal epidemiology research.

\section{Methods}

\section{Participants}

Seventy-three participants, aged 45 to 84 years, who were able to complete activities of daily living without assistance, were recruited via posted flyers, e-mail announcements, and word of mouth from Columbia, SC and surrounding communities. During a brief enrollment telephone call, individuals with health conditions that would contraindicate study participation were identified and were required to obtain written consent from their physician before study participation. During that same phone call, participants were asked about their current PA levels by responding to questions from the Behavioral Risk Factor Surveillance System. ${ }^{14}$ Participants were mailed documents that included standardized instructions to follow before the laboratory visit (eg, no eating or drinking within 4 hours of the test, no consuming caffeine or alcohol within 12 hours of the test, and no exercising within 6 hours of the test).

\section{Protocol}

Testing was completed during a single 2-hour visit to the University of South Carolina Clinical Research Center. Upon arrival to the testing site, an overview of the study protocol was provided to each participant, who then gave written informed consent in accordance with procedures approved by the University of South Carolina Office of Research Compliance. Body mass $(\mathrm{kg})$ and body composition (percent fat mass) were determined using a leg-to-leg bioelectrical impedance analysis (BIA) system (Tanita 300A, Tokyo, Japan). Equations currently used in the Tanita 300A BIA system have been generalized to allow fat-free mass estimates from adults varying widely in body composition and age. ${ }^{15,16}$ The standard error of estimate for percent body fat is approximately $3.5 \%,{ }^{17}$ which is similar to that observed for skinfold measurements. ${ }^{18}$ Strong correlations ( $r=.91$ to .93 ) have also been observed in mean fat-free mass predicted by BIA and hydrostatic weighing. ${ }^{16,19}$ The measurement of body height $(\mathrm{cm})$ allowed for the calculation and classification of body mass index $\left(\mathrm{BMI}, \mathrm{kg} \cdot \mathrm{m}^{2}\right) .{ }^{20}$

During the protocol each participant completed 8 simulated physical activities of daily living (Table 1). The same verbal and visual instructions were given to each participant, and the tasks were always performed in the same sequence. The activities were supine resting (13 minutes), TV viewing (4 minutes), card playing (4 minutes), sweeping (5 minutes), vacuuming (5 minutes), treadmill walking at $2.5 \mathrm{mph}$ (5 minutes), treadmill walking at $3.0 \mathrm{mph}$ (5 minutes), and stair stepping (5 minutes). Supine resting was used to determine resting metabolic rate. TV viewing and card playing represented sitting activities, sweeping and vacuuming simulated household tasks that use upper and lower body limbs, and the final 3 tasks represented locomotive activities. During the last minute of each task, participants reported their rating of perceived exertion (RPE) using the 6 to 20 point Borg scale. ${ }^{21}$ All tasks were separated with 1 to 3 minutes of seated rest. Before being fitted with any instrumentation and formally beginning the testing protocol, each participant completed a 3-minute session on the treadmill at speeds of $2.5 \mathrm{mph}$ and $3.5 \mathrm{mph}$ to become familiar with that specific activity. Approximately 30 minutes of seated rest elapsed between the treadmill orientation and start of the supine resting period.

\section{Metabolic Measurement Instrumentation}

The participant was fitted with a specially designed harness that carried a portable metabolic measurement system (Cosmed Model K4b2, Rome, Italy). The portable metabolic measurement system weighs $1.6 \mathrm{~kg}$, including the battery and harness. The system was used to determine energy expenditure from oxygen uptake $\left(\mathrm{VO}_{2}\right)$ and carbon dioxide production $\left(\mathrm{VCO}_{2}\right)$ on a breath-by-breath basis during all activities. The Cosmed K4b2 has been shown to be a valid device compared with the Douglas bag technique during cycle ergometry. ${ }^{22}$ The gas analyzers and airflow turbine were calibrated before each testing session according to the manufacturer's instructions. During testing, a mask was tightly secured over the mouth and nose to eliminate the loss of any expired air into the room.

The Actical activity monitor (Mini Mitter Respironics, Inc., Bend, OR) used for this study is water resistant, lightweight $(17 \mathrm{~g})$, small $\left(2.8 \times 2.7 \times 1.0 \mathrm{~cm}^{3}\right)$, and has a data storage capacity of 64,800 data points that will saturate after 44 days of continuous measurement using 1-min recording intervals (epochs). The monitor was initialized and downloaded before each test using a serial port computer interface, with the resulting data exportable as text files. The Actical uses a single internal "omnidirectional" accelerometer that senses motion in all directions, but is most sensitive within a single plane. It detects low frequency $(0.5$ to $3.2 \mathrm{~Hz}) \mathrm{G}$-forces $(0.05$ to $2.0 \mathrm{~Hz}$ ) common to human movement and generates an analog voltage signal that is filtered and amplified before being digitized by an A-to-D converter at $32 \mathrm{~Hz}$. In this study, the digitized values were summed over 1-minute epochs. The actual numbers stored by the Actical are proportional to the magnitude and duration of the sensed accelerations. ${ }^{3}$ The Actical was secured to an elastic belt and positioned snugly over the right iliac crest by research staff with consistent orientation as recommended by the manufacturer.

\section{Analysis}

Participants were divided into 3 groups for analysis based on body composition and age. Group 1 was comprised of 29 nonobese individuals age 45 to 64 years with both 
Table 1 Durations and Descriptions of Activities Performed by Participants

\begin{tabular}{|c|c|c|c|}
\hline Activity type & Name of activity & $\begin{array}{c}\text { Time } \\
\text { (minutes) }\end{array}$ & Description of activity \\
\hline Resting & Supine resting & 13 & $\begin{array}{l}\text { Participants rested on a bed in the supine position in a thermal- } \\
\text { controlled room with instructions to limit talking and bodily } \\
\text { movement, and not fall asleep. }\end{array}$ \\
\hline \multirow[t]{2}{*}{ Sitting } & Television viewing & 4 & $\begin{array}{l}\text { Participants were instructed to sit in a chair facing the television } \\
\text { and view a local public station program. }\end{array}$ \\
\hline & Card playing & 4 & $\begin{array}{l}\text { While sitting in a chair in front of a desk, participants played } \\
\text { either a standard card game or sorted cards in suits for the dura- } \\
\text { tion of the task. }\end{array}$ \\
\hline \multirow[t]{2}{*}{ Household cleaning } & Sweeping & 5 & $\begin{array}{l}\text { Confetti, representing dirt, was placed on one side of a large } \\
\text { exercise mat }\left(2.5 \mathrm{M} \times 1.2 \mathrm{M} / 8.2^{\prime} \times 3.9^{\prime}\right) \text {. Participants used a stan- } \\
\text { dard indoor broom }(\mathrm{kg} / \mathrm{lb}) \text { to sweep the confetti from one side of } \\
\text { the mat to the other while moving slowly down the mat. When } \\
\text { finished moving down the mat, participants turned around and } \\
\text { repeated the task. }\end{array}$ \\
\hline & Vacuuming & 5 & $\begin{array}{l}\text { Participants vacuumed a carpeted area }(6 \times 3.3 \mathrm{M} / 19.6 \text { ' } \times 10.8 \text { ' }) \text { in } \\
\text { multiple directions using an upright, lightweight vacuum }(6.6 \\
\mathrm{kg} / 14.5 \mathrm{lb}) \text { and moving slowly, but continuously for the duration } \\
\text { of the task. }\end{array}$ \\
\hline \multirow[t]{2}{*}{ Locomotion } & Slow treadmill walking & 5 & $\begin{array}{l}\text { Treadmill speed was set at } 2.5 \mathrm{mph}\left(67 \mathrm{~m} \cdot \mathrm{min}^{-1}\right) \text { at a level }(0 \%) \\
\text { grade. Participants were instructed not to hold on to the side rail } \\
\text { unless they felt they were going to lose their balance. }\end{array}$ \\
\hline & Brisk treadmill walking & 5 & $\begin{array}{l}\text { Treadmill speed was set at } 3.5 \mathrm{mph}\left(94 \mathrm{~m} \cdot \mathrm{min}^{-1}\right) \text { at a level }(0 \%) \\
\text { grade. Participants were instructed not to hold on to the side rail } \\
\text { unless they felt they were going to lose their balance. }\end{array}$ \\
\hline
\end{tabular}

a BMI $<30 \mathrm{~kg} \cdot \mathrm{m}^{-2}$ and a percent body fat $<32 \%$ for females or $<25 \%$ for males. Group 2 was comprised of 21 participants age 45 to 64 years who were obese (BMI $\geq 30 \mathrm{~kg} \cdot \mathrm{m}^{-2}$ and percent body fat $\geq 32 \%$ for females or $\geq 25 \%$ for males). The 23 participants in Group 3 were classified by age alone ( $\geq 65$ years), regardless of body composition ( $\mathrm{N}=19$ nonobese and $\mathrm{N}=4$ obese).

Accelerometer and metabolic data were averaged over the final 2 minutes of each task. Three obese 45 to 64 year old participants and 11 participants over 65 years of age could only complete 3 to 4 minutes of treadmill walking at $3.5 \mathrm{mph}$. Similarly, 1 nonobese 45 to 64 year old participant, 12 obese 45 to 64 year old participants, and $15 \geq 65$ year old participants completed 3 to 4 minutes of stair stepping. In these instances, the last 2 minutes of data were used if the $\mathrm{VO}_{2}$ and $\mathrm{VCO}_{2}$ were within $100 \mathrm{ml}$ of one another indicating a steady state had been attained. If these criteria were not met (ie, $\mathrm{N}=1$ to 4 participants from the obese 45 to 64 year old and $\geq 65$ year old groups for either $3.5 \mathrm{mph}$ walking or stair stepping), the data for that activity were not included in the analysis. Energy expenditure for supine resting (EESR) was determined as an average over the last $5 \mathrm{~min}$.

Activity energy expenditure (AEE, $\mathrm{kcal} \cdot \mathrm{kg}^{-1} \cdot \mathrm{min}^{-1}$ ) was defined as the relative rate of energy expenditure above EESR:

$$
\mathrm{AEE}_{\mathrm{ij}}=\left(\mathrm{EE}_{\mathrm{ij}}-\mathrm{EESR}_{\mathrm{j}}\right) / \mathrm{MT}_{(\mathrm{j})},
$$

where $\mathrm{AEE}_{\mathrm{i}}\left(\mathrm{kcal} \cdot \mathrm{kg}^{-1} \cdot \mathrm{min}^{-1}\right)$ was the computed value for the $i$ th activity and participant $j$. Values for $\mathrm{AEE}_{\mathrm{ij}}$ were derived from the corresponding $i$ th mean absolute energy expenditure $\left(\mathrm{EE}_{\mathrm{i}}, \mathrm{kcal} \cdot \mathrm{min}^{-1}\right)$ for participant $j$, the computed EESR $\mathrm{E}_{\mathrm{j}}$ for participant $j$, and the total mass of participant $j$ and equipment $\left(\mathrm{M}_{\mathrm{T}}(\mathrm{j}), \mathrm{kg}\right)$. Values for $\mathrm{EE}_{\mathrm{i}}$ were calculated using Weir's equation: ${ }^{23}$

$$
\mathrm{EEi}=3.9 \times \mathrm{VO}_{2(\mathrm{i})}+1.1 \times \mathrm{VCO}_{2(\mathrm{i})},
$$

where $\mathrm{VO}_{2(\mathrm{i})}\left(\mathrm{L} \cdot \mathrm{min}^{-1}\right)$ and $\mathrm{VCO}_{2(\mathrm{i})}\left(\mathrm{L} \cdot \mathrm{min}^{-1}\right)$ were the average $\mathrm{VO}_{2}$ and $\mathrm{VCO}_{2}$ values, respectively, corresponding to the $i$ th activity. METs were calculated using only $\mathrm{VO}_{2}$ and body weight, defined as $\mathrm{VO}_{2}$ divided by body weight divided by a standard $3.5 \mathrm{~mL} / \mathrm{kg}$-min. 
An accelerometer does not directly measure energy expenditure, rather it records vector quantities of vertical force in units referred to as activity counts (AC). Higher AC result from greater vertical forces and thus reflect higher intensity PA. Therefore, equations can be developed to predict METs from AC data. Actical $\mathrm{AC}$ data obtained from the hip position were plotted against METs obtained from indirect calorimetry $\left(\mathrm{VO}_{2}\right)$. Nonlinear regression was used to fit METs to AC. The seated activities (TV viewing and card sorting) were omitted from this procedure since the recorded AC were almost always 0 . The stair stepping activity was also not used in this procedure because accelerometer patterns from level walking at an average pace remain essentially unchanged during walking up stairs or hills despite the increased energy cost. ${ }^{24,25}$ Furthermore, inclusion of the household and walking activities yielded the highest $r^{2}$ value and the smallest mean difference between actual and predicted METs.

To initially confirm the suitability of hip AC as a predictor of MVPA, receiver operating characteristic (ROC) curves were visually examined, and the area under those curves computed by subgroup and for the groups combined. The very large area under each ROC curve ( 0.93 to 0.97 depending on group) indicated that setting an appropriate AC cut-point could be expected to categorize PA levels with acceptable sensitivity and specificity. Raw data scatter plots of METs versus AC exhibited various degrees of curvature in the relationship, varying by group, indicative of the need to introduce transform or nonlinear terms into regression models.

The nonlinear regression equation used included, in addition to the usual intercept and slope, a power or exponent parameter on the independent variable AC. This is a more general approach to account for curvature in the METs-AC relationship than the commonly used logarithm or square-root transformation, and it has been used in previous accelerometer studies. ${ }^{26-30}$ The METs value for the $i$ th subject is fitted to the AC value by

$$
\operatorname{METS}_{i}=\beta_{0}+\beta_{1} A C_{i}^{\beta_{2}}+\varepsilon_{i},
$$

with the 3 parameters estimated using PROC NLIN of the SAS System. Although the use of a power parameter was not required for achieving an acceptable r-squared value in the regressions, it eliminated the tendency of linear regression to overestimate METs at higher AC values.

For comparison, both nonlinear and linear regression models were estimated by subgroup and for the entire study sample. An additional model was created for the entire sample with METs predicted by hip AC along with covariates for age and body composition (both BMI and percent body fat were included). For every model examined, a 3 MET AC cut-point was computed and the proportion of correct classification compared across models using METs from the metabolic cart as criterion. Selection of the preferred models was based on the idea of using the simplest model possible without resulting in substantially worse MVPA classification accuracy.
To assess classification accuracy, 2-by-2 tables of PA intensity less or greater than 3 METs versus hip AC less or greater than each particular cut-point were constructed. From these tables, an overall percentage of correct classification was computed along with false-positive and falsenegative percentages leading to the customary measures of sensitivity, specificity, and/or positive predictive value.

\section{Results}

The sample comprised 29, 21, and 23 participants in the nonobese 45 to 64 year, obese 45 to 64 year, and $\geq 65$ year old groups, respectively. Demographic characteristics for each group are provided in Table 2. Obese 45 to 64 year old participants had significantly higher body weight, BMI, and percent body fat compared with their nonobese counterparts and with participants $\geq 65$ years of age.

Mean values for indirect calorimetry, RPE, and Actical output variables are given in Table 3 . The EESR was less than 1 MET for each group, with the obese 45 to 64 year old participants exhibiting the lowest mean value $(0.72 \pm 0.17$ METs). Actical AC for each group were at or near 0 for supine rest, TV viewing, and card sorting, indicating minimal vertical acceleration forces at the hip during these activities. The $\geq 65$ year old participants tended to record lower AC during sweeping and vacuuming activity than the 2 younger age groups despite having similar, if not higher, levels of $\mathrm{VO}_{2}$ during the standardized tasks. Walking on the treadmill at 2.5 $\mathrm{mph}$ and $3.5 \mathrm{mph}$ resulted in very similar $\mathrm{AC}$ across the 3 groups; however, AEE levels were variable. The obese 45 to 64 year old participants tended to exhibit the lowest $\mathrm{VO}_{2}$ and $\mathrm{AEE}$ values for each activity.

The laboratory-simulated household and locomotive activities did not elicit an expenditure $\geq 6$ METs in any of the participants. Walking at $3.5 \mathrm{mph}$ and stair stepping resulted in group means of 4.2 to 4.6 METs and 4.5 to 5.0 METs, respectively (Table 3 ). Thus, it was not possible to confidently determine an AC cut-point equivalent to $\geq 6$ METs.

Table 4 contains the regression parameters (intercept, slope and exponent) for the METs-AC relationship within each age and body composition subgroup and for the entire sample combined. Group-specific models achieved slightly higher $r$-squared values ( 0.74 to 0.80$)$ than the single overall model $(0.72)$, but in all cases the predictive value of hip AC for METs was good. The 3 MET AC cut-point value for the $\geq 65$ year old group diverged significantly from the results for the 2 younger groups and the overall sample. This difference was primarily due to the greater curvature of the AC-METs relationship in $\geq 65$ year old group, reflected in an exponent parameter of 0.347 compared with approximately 0.81 in the younger subgroups.

Taking all 4 activities $(2.5 \mathrm{mph}$ and $3.5 \mathrm{mph}$ walking, sweeping, vacuuming) together, the individual minutes of observation were correctly categorized as less or greater than 3 METs $84.0 \%$ of the time when 1 overall AC 


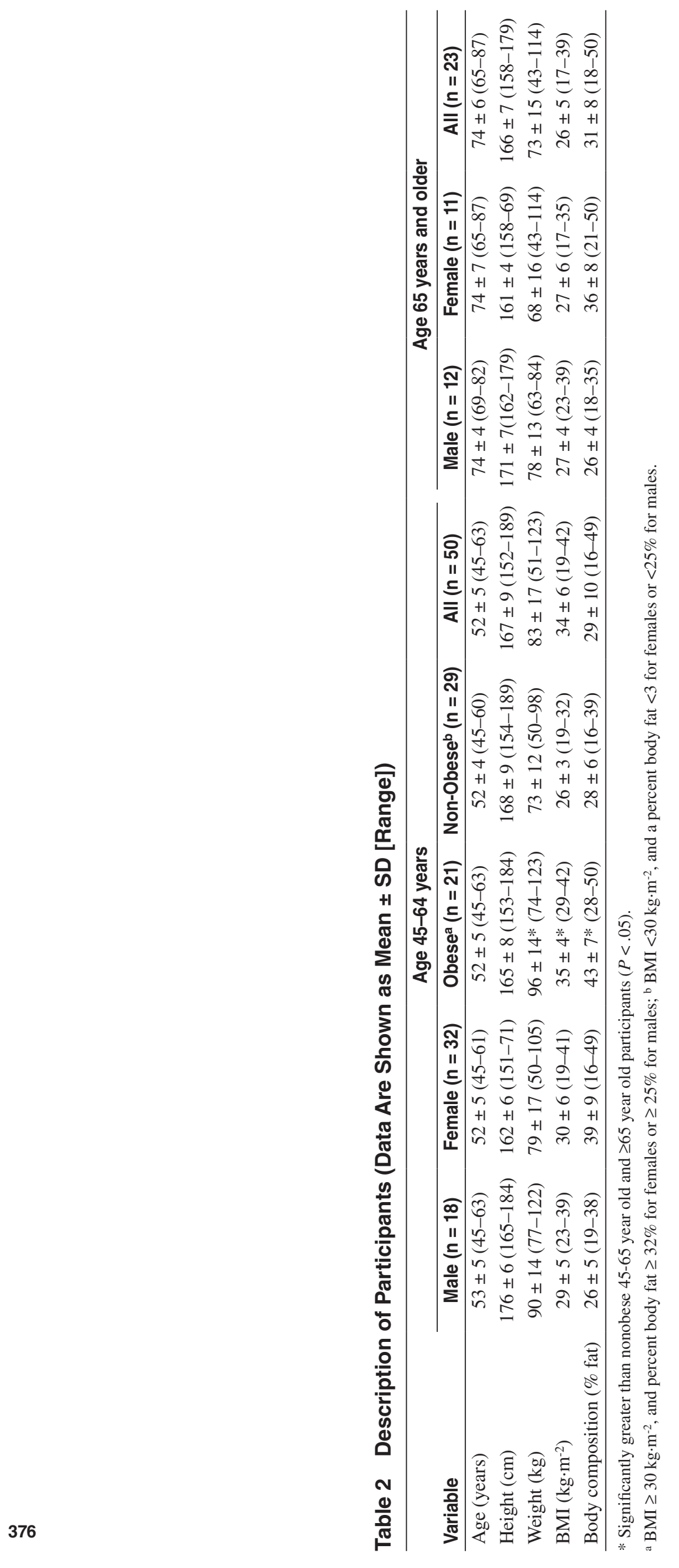




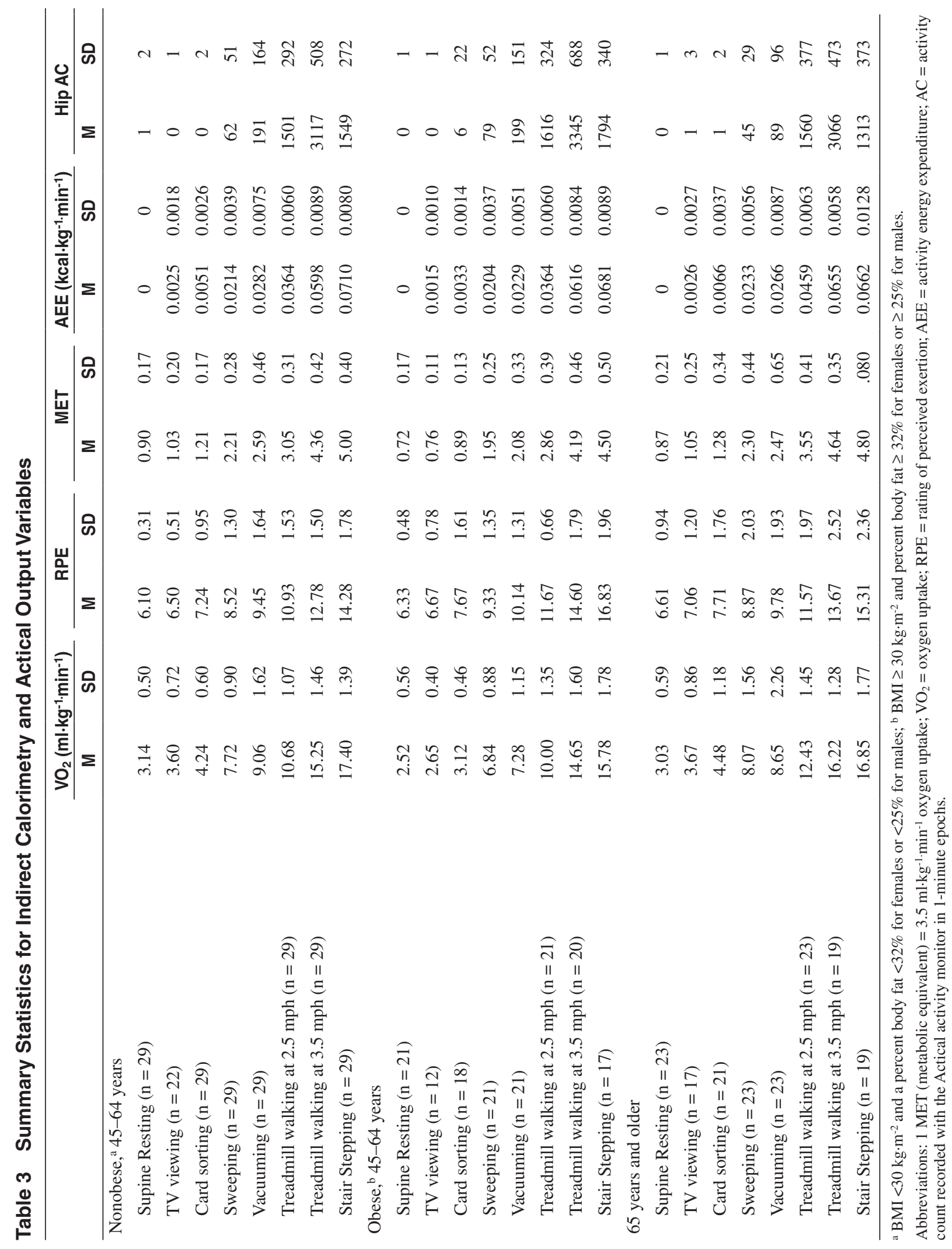


Table 4 Activity Count (AC) Cut Points Obtained From the Actical at the Hip Position Corresponding to Moderate Intensity (3 METs*) PA

\begin{tabular}{ll}
\hline Group & Equations \\
\hline Nonobese, ${ }^{\text {a }} 45-64$ years & AEE $\left(\mathrm{kcal} \cdot \mathrm{kg}^{-1} \cdot \mathrm{min}^{-1}\right)=0.0220+(4.460 \mathrm{E}-5) \times \mathrm{AC}^{0.828}\left(r^{2}=.74 ; \mathrm{SEE}=.0083\right)$ \\
Obese, ${ }^{\mathrm{b}} 45-64$ years & $\mathrm{AEE}\left(\mathrm{kcal} \cdot \mathrm{kg}^{-1} \cdot \mathrm{min}^{-1}\right)=0.0194+(3.362 \mathrm{E}-5) \times \mathrm{AC}^{0.868}\left(r^{2}=.79 ; \mathrm{SEE}=.0079\right)$ \\
65 years and older & $\mathrm{AEE}\left(\mathrm{kcal} \cdot \mathrm{kg}^{-1} \cdot \mathrm{min}^{-1}\right)=0.0175+(1.160 \mathrm{E}-3) \times \mathrm{AC}^{0.454}\left(r^{2}=.84 ; \mathrm{SEE}=.0072\right)$ \\
Combined & $\mathrm{AEE}\left(\mathrm{kcal} \cdot \mathrm{kg}^{-1} \cdot \mathrm{min}^{-1}\right)=0.0210+(8.574 \mathrm{E}-5) \times \mathrm{AC}^{0.755}\left(r^{2}=.76 ; \mathrm{SEE}=.0084\right)$ \\
\hline
\end{tabular}

$* 1$ MET (metabolic equivalent) $=3.5 \mathrm{ml} \cdot \mathrm{kg}^{-1} \cdot \mathrm{min}^{-1}$ oxygen uptake.

${ }^{\text {a }} \mathrm{BMI}<30 \mathrm{~kg} \cdot \mathrm{m}^{-2}$ and a percent body fat $<32 \%$ for females or $<25 \%$ for males.

${ }^{\mathrm{b}} \mathrm{BMI} \geq 30 \mathrm{~kg} \cdot \mathrm{m}^{-2}$ and percent body fat $\geq 32 \%$ for females or $\geq 25 \%$ for males.

cut-point of 1065 was applied. Use of 3 group-specific $\mathrm{AC}$ cut-points $(1107,1634$, and 431) improved this accuracy only trivially to $84.5 \%$, with a slight decrease in the percentage of false-positive results from $19.1 \%$ to $17.9 \%$, and a very small increase from $11.6 \%$ to $12.0 \%$ in the rate of false-negatives.

Using a single AC cut-point of 1065 resulted in accuracy of $98.6 \%, 89.7 \%$, and $100.0 \%$ classification accuracy for sweeping, vacuuming and $3.5 \mathrm{mph}$ walking, respectively. Only the $2.5 \mathrm{mph}$ walking task produced numerous minutes of observation in which the AEE was near the 3 METs threshold (Table 3). Thus, this task presented the greatest challenge to the ability of hip AC to predict PA intensity and accuracy for this task was relatively poor. Using a single cut-point of 1065 produced only $49.3 \%$ correct classification of minutes as less or greater than 3 METs for the 2.5 mph walking task. No meaningful improvement was noted with subgroup AC cut-points which resulted in $50.7 \%$ correct classification. This misclassification was almost entirely in the form of false positives meaning, of the minutes for which measured METs were less than 3.0, a single AC cut-point of 1065 predicted moderate intensity $97.0 \%$ of the time. Subgroup AC cut-points only slightly decreased misclassification to $91.0 \%$.

Regression equations to predict AEE from Actical $\mathrm{AC}$ are provided in Table 5. These AEE-AC relationships resulted in very strong correlations $\left(r^{2}=.74\right.$ to .82 , SEE $=.0078$ to $.0082 \mathrm{kcal} / \mathrm{kg} / \mathrm{min}$ ) within each group.

\section{Discussion}

The purpose of the current study was to develop an AC cut-point to differentiate between light and moderateintensity PA in middle-aged and older adults, using the Actical activity monitor worn at the hip. We grouped study participants according to both their BMI and percent body fat to better account for the influence that body composition might have on the 3 METs AC cut-point. Findings from this study indicate that, although the AC cut-points associated with moderate-intensity activity varied somewhat among the 3 groups, a common AC cut-point of 1065 yielded similar accuracy for detecting an activity as less or greater than 3 METs in our sample of people $\geq 45$ years of age regardless of age and body composition. Even though a common AC cut-point may be suitable for persons $\geq 45$ years of age, the derived AC cut-point of 1065 is substantially higher than the AC cut-point of 281 previously applied to younger persons (mean \pm SD age $34 \pm 8$ years for men and $39 \pm 10$ years for women) wearing the Actical. ${ }^{3}$ These results verify the importance of developing population-specific AC cut-points for individual accelerometers. ${ }^{13,29,30}$

Activity count cut-points are essential to monitoring the time spent in PA of varying intensity, duration and frequency, in determining which persons meet or do not meet PA recommendations, and in estimating overall energy expenditure. Misapplying AC cut-points could result in misclassification of persons with regard to activity status and miscalculation of key PA variables related to a multitude of health outcomes. ${ }^{2}$ To our knowledge, this is the first study to show that Actical AC cut-points are affected by age. If the notably lower 3 METs AC cut-point of 281 were applied in studies of free-living adults age $\geq 45$ years, this would result in dramatically more minutes of moderate intensity PA being identified than are actually achieved.

Although a cross-validation component was not included in the current study, the 281 and 1065 AC cutpoints were applied to 7-day accelerometer data obtained from 71 midlife and older adults who wore an Actical under free-living conditions (unpublished data). Applying the 1065 AC cut-point resulted in $25 \pm 18$ minutes per week of MVPA which corresponds closely to the amount of objectively measured MVPA observed in other adult populations..$^{11,31,32}$ Using the $281 \mathrm{AC}$ cut-point equated to $157 \pm 65$ minutes per week of MVPA, or nearly 6 times more than noted with the 1065 cut-point. These results verify the need to make an age-related adjustment with middle-aged and older adults wearing the Actical by applying an AC cut-point of 1065.

The need to use higher than previously established Actical AC cut-points to distinguish light and moderate intensity PA in overweight and obese adults has been previously suggested. ${ }^{33}$ The potential exists for 
Table 5 Equations to Predict AEE (kcal $\left.\cdot \mathrm{kg}^{-1} \cdot \mathrm{min}^{-1}\right)$ Using Actical Activity Count (AC) Data Obtained From the Hip Position*

\begin{tabular}{ll}
\hline Group & Equations \\
\hline Nonobese $^{\text {a }}$ 45-64 years & AEE $\left(\mathrm{kcal} \cdot \mathrm{kg}^{-1} \cdot \mathrm{min}^{-1}\right)=0.0220+(4.460 \mathrm{E}-5) \times \mathrm{AC}^{0.828}\left(r^{2}=.74 ; \mathrm{SEE}=.0083\right)$ \\
Obese, ${ }^{\mathrm{b}} 45-64$ years & AEE $\left(\mathrm{kcal} \cdot \mathrm{kg}^{-1} \cdot \mathrm{min}^{-1}\right)=0.0194+(3.362 \mathrm{E}-5) \times \mathrm{AC}^{0.868}\left(r^{2}=.79 ; \mathrm{SEE}=.0079\right)$ \\
65 years and older & AEE $\left(\mathrm{kcal} \cdot \mathrm{kg}^{-1} \cdot \mathrm{min}^{-1}\right)=0.0175+(1.160 \mathrm{E}-3) \times \mathrm{AC}^{0.454}\left(r^{2}=.84 ;\right.$ SEE $\left.=.0072\right)$ \\
Combined & AEE $\left(\mathrm{kcal} \cdot \mathrm{kg}^{-1} \cdot \mathrm{min}^{-1}\right)=0.0210+(8.574 \mathrm{E}-5) \times \mathrm{AC}^{0.755}\left(r^{2}=.76 ; \mathrm{SEE}=.0084\right)$ \\
\hline
\end{tabular}

* Activities included sweeping, vacuuming, treadmill walking at $2.5 \mathrm{mph}$, treadmill walking at $3.5 \mathrm{mph}$.

Abbreviations: AEE = activity energy expenditure.

${ }^{\text {a }} \mathrm{BMI}<30 \mathrm{~kg} \cdot \mathrm{m}^{-2}$ and a percent body fat $<32 \%$ for females or $<25 \%$ for males; ${ }^{\text {b }} \mathrm{BMI} \geq 30 \mathrm{~kg} \cdot \mathrm{m}^{-2}$ and percent body fat $\geq 32 \%$ for females or $\geq$ $25 \%$ for males.

weight-related changes in basal metabolic rate and fat free mass contributing to alterations in the energy expenditure associated with any given PA and related accelerometer AC. ${ }^{12}$ However, our results did not indicate the need for a body composition-related adjustment with middle-aged and older adults. The individual group and collective AC cut-points derived from regression models using BMI and percent body fat did not improve the accuracy of determining light and moderate intensity PA. The additional complication of computing individual-specific AC cut-points taking into account age and body composition is not justified given the lack of improved results, at least not in studies where categorizing minutes of activity as less or greater than 3 METs is the goal. The middle-age obese group had a mean BMI of $35 \mathrm{~kg} \cdot \mathrm{m}^{-2}$ and $43 \%$ body fat, and perhaps different results would have been found if persons with even greater degrees of obesity had been included.

Although household and locomotive activities commonly performed by middle-aged and older adults were selected for inclusion in this study, the laboratory-simulated activities did not require an energy expenditure $\geq 6$ METs. Energy expenditures demonstrated during walking at $3.5 \mathrm{mph}$ corresponded to 4.2 to 4.6 METs and 12.8 to 14.6 RPE values (Table 3), demonstrating that walking at this pace was of moderate intensity, both metabolically and perceptually. A recent study also noted the inability to include vigorous intensity PA in an accelerometer validation protocol with older adults. ${ }^{7}$ These investigators pointed out that although some older persons do engage in high intensity PA, they are not likely to be representative of the population. Indeed, recent accelerometer studies reveal that $>95 \%$ of middle-aged and older adults engage in less than 5 minutes of vigorous intensity PA in a typical week. ${ }^{11,31,32}$ Thus, the most vital AC cut-point for these adult populations is one that delineates between light and moderate intensity PA (ie, 3 METs).

As mentioned previously, despite following a nearly identical protocol implemented with younger adults, the metabolic costs associated with the selected laboratorybased activities were lower than previously reported. As noted in Table 3, the AC associated with the simulated household activities were also lower than observed before. ${ }^{3}$ Accordingly, many of the participants in each group exhibited AC during household activities that fell below 50 counts $\cdot \mathrm{min}^{-1}$, the formerly defined value denoting the AC cut-point between sedentary and light intensity activity. The present findings indicate that for middle-aged and older adults, the AC cut-point separating sedentary and light intensity activities should be lowered to 25 counts $\cdot \mathrm{min}^{-1}$. The ability to accurately delineate between sedentary and light intensity PA is becoming more vital as sedentary pursuits represent a unique aspect of human behavior and emerging research indicates that time spent in sedentary activities is strongly related to the risk for developing dyslipidemia, obesity, type 2 diabetes, hypertension, metabolic syndrome, and CVD. ${ }^{34-36}$ We recommend additional studies to verify the Actical AC cut-point(s) that most accurately identifies time spent being sedentary in various age groups.

In practice, the developed 3 METs AC cut-point may provide useful prediction of various PA variables for groups of middle-aged and older adults, but the tracking of individuals may still involve some error. ${ }^{2}$ Accelerometers are relatively insensitive to nonweight bearing activities (such as cycling), lifting heavy objects, and surface incline/decline during locomotion. ${ }^{37}$ Therefore, it is not reasonable to expect any accelerometer or data processing algorithm using generalized AC cut-points to ever accurately detect intensity and duration for all activities for all people. ${ }^{3,30,38,39}$ However, walking and other activities performed in an upright posture are vastly common among the populations we studied. ${ }^{40}$ Within the context of these issues, the current study did observe individual variability in prediction accuracy for the laboratory-based activities, with much less variability for household activities and walking at $3.5 \mathrm{mph}$ as compared with walking at $2.5 \mathrm{mph}$.

The difficulty with using hip AC to distinguish between PA only slightly less or greater than 3 METs intensity during the $2.5 \mathrm{mph}$ walking activity is, in one sense, an artifact of that particular choice of laboratory activity. It is probable that free-living activity patterns will only infrequently cluster at or near 3 METs. However, even when applied to a large population, the use of an overall 3 METs AC cut-point may tend to "credit" 
minutes of MVPA for certain locomotive activities regardless of whether the actual energy expenditure is slightly lower or higher than 3.0 METS.

One alternative would be to select a threshold higher (eg, 4 METS) or lower (eg, 2.5 METS) as the bound of "moderate" activity for middle-aged and older adults. But, it seems likely that any reasonable choice of threshold would cause similar errors in classification for some common categories of PA. It may be necessary to simply accept, as a fundamental limitation of accelerometer technology, that population-based AC cut-points will not be error-free in identifying a definitive threshold between light and moderate intensity PA. ${ }^{13}$

The current project followed similar laboratory methods as did an earlier Actical validation study involving younger adults. ${ }^{3}$ The main difference in the analytical approach was modeling METs directly from Actical AC rather than predicting AEE from $\mathrm{AC}$, and METs from AEE in a 2-step process. The current analysis also used a power function of $\mathrm{AC}$, which has previously proven effective in accelerometer validation studies, ${ }^{26-30}$ rather than a piecewise linear equation. These differences aside, we believe that the results are meaningful and comparable. The findings reiterate the importance of deriving accelerometer data processing components (ie, intensity cutpoints and accelerometer-to-AEE conversion algorithms) that are population-specific, especially pertaining to age. In nonobese and obese middle-aged and older adults, a standard AC cut-point of 1065 yielded similar accuracy in predicting light and moderate intensity activity, and can be used to monitor the PA patterns of large groups of such persons.

\section{Acknowledgments}

The authors are grateful to Chris Davis and Ellen Henderson for their assistance in collecting the data. This study was supported by a grant from Mini Mitter Respironics.

\section{References}

1. Troiano RP. Translating accelerometer counts into energy expenditure: advancing the quest. J Appl Physiol. 2006;100:1107-1108.

2. Matthews CE. Calibration of accelerometer output for adults. Med Sci Sports Exerc. 2005;37(11, Suppl):S512S522.

3. Heil DP. Predicting activity energy expenditure using the Actical activity monitor. Res Q Exerc Sport. 2006;77(1):64 80 .

4. Troiano RP. A timely meeting: objective measurement of physical activity. Med Sci Sports Exerc. 2005;37(11, Suppl):S487-S489.

5. Crouter SE, Clowers KG, Bassett DR, Jr. A novel method for using accelerometer data to predict energy expenditure. J Appl Physiol. 2006;100:1324-1331.

6. Macfarlane D, Lee C, Ho E, Chan KL, Chan D. Convergent validity of six methods to assess physical activity in daily life. J Appl Physiol. 2006;101:1328-1334.
7. Copeland JL, Esliger DW. Accelerometer assessment of physical activity in active, healthy older adults. J Aging Phys Act. 2009;17(1):17-30.

8. Westerterp KR. Physical activity as determinant of daily energy expenditure. Physiol Behav. 2008;93(4-5):10391043.

9. Hagstromer M, Oja P, Sjostrom M. Physical activity and inactivity in an adult population assessed by accelerometry. Med Sci Sports Exerc. 2007;39(9):1502-1508.

10. Rowe DA, Kemble CD, Robinson TS, Mahar MT. Daily walking in older adults: day-to-day variability and crieterion-referenced validity of total daily step counts. $J$ Phys Act Health. 2007;4(4):434-446.

11. Berrigan D, Troiano R. Physical activity measured by accelerometry in the National Health and Nutrition Examination Survey 2003-04. Available at: http://www. activelivingresearch.org/alr/files/Berrigan_Accelerometry_2007.pdf. Accessed December 18, 2007

12. Murphy SL. Review of physical activity measurement using accelerometers in older adults: considerations for research design and conduct. Prev Med. 2009;48(2):108114.

13. Welk GJ. Principles of design and analyses for the calibration of accelerometry-based activity monitors. Med Sci Sports Exerc. 2005;37(11, Suppl):S501-S511.

14. Centers for Disease Control and Prevention (CDC). Behavioral risk factor surveillance system questionnaire. Atlanta, GA: U.S. Department of Health and Human Services, Centers for Disease Prevention and Control; 2006.

15. Utter A, Nieman D, Ward A, Butterworth D. Use of the leg-to-leg bioelectrical impedance method in assessing body composition changes in obese women. Am J Clin Nutr. 1999;69:603-607.

16. Utter A, Nieman D, Mulford G, et al. Evaluation of legto-leg BIA in assessing body composition of high-school wrestlers. Med Sci Sports Exerc. 2005;37(8):1395-1400.

17. Stolarczyk L, Heyward V, Van Loan M, Hicks V, Wilson W, Reano L. The fatness-specific bioelectric impedance analysis equations of Segal et al: are they generalizable and practical? Am J Clin Nutr. 1997;66:8-17.

18. Heyward B, Wagner D. Applied body composition assessment. 2nd ed. Champaign, IL: Human Kinetics; 2004.

19. Cable A, Nieman D, Austin M, Hogen E, Utter A. Validity of leg-to-leg bioelectrical impedance measurement in males. J Sports Med Phys Fitness. 2001;41:411-414.

20. National Heart, Lung, and Blood Institute. Clinical guidelines on the identification, evaluation, and treatment of overweight and obesity in adults: the evidence report. Bethesda, MD: National Institutes of Health, National Heart, Lung, and Blood Institute; 1998.

21. Borg G. Psychophysical bases of perceived exertion. Med Sci Sports Exerc. 1982;14(5):377-387.

22. McLaughlin JE, King GA, Howley ET, Bassett DR, Jr, Ainsworth BE. Validation of the COSMED K4b2 portable metabolic system. Int J Sports Med. 2001;22:280-284.

23. Weir JBdV. New methods for calculating metabolic rate with special reference to protein metabolism. J Physiol. 1949;109:1-9.

24. Bassett D. Validity and reliability issues in objective monitoring of physical activity. Res $Q$ Exerc Sport. 2000;71:S30-S36.

25. Welk GJ, Morss G, Church T, Differding J. Laboratory calibration of the Biotrainer and Actitrac activity monitors. Med Sci Sports Exerc. 2002;34(5):S140. 
26. Chen KY, Acra SA, Majchrzak K, et al. Predicting energy expenditure of physical activity using hip- and rest-worn accelerometers. Diabetes Technol Ther. 2003;5(6):10231033.

27. Chen KY, Bassett D. The technology of accelerometrybased activity monitors: current and future. Med Sci Sports Exerc. 2005;37(11):S490-S500.

28. Chen KY, Sun M. Improving energy expenditure estimation by using a triaxial accelerometer. J Appl Physiol. 1997;83:2112-2122.

29. Pfeiffer K, McIver K, Dowda M, Almeida M, Pate R. Validation and calibration of the Actical accelerometer in preschool children. Med Sci Sports Exerc. 2006;38:152-157.

30. Puyau MR, Adolph AL, Vohra FA, Zakeri I, Butte NF. Prediction of activity energy expenditure using accelerometers in children. Med Sci Sports Exerc. 2004;36(9):1625-1631.

31. Hagstromer M, Oja P, Sjostrom M. Physical activity and inactivity in an adult population assessed by accelerometry. Med Sci Sports Exerc. 2007;39:1502-1508.

32. Rowe DA, Kemble CD, Robinson TS, Mahar MT. Daily walking in older adults: day-to-day variability and criterion-referenced validity of total daily steps. $J$ Phys Act Health. 2007;4:434-446.

33. Heil D, Hogan P, Whitt-Glover M. The influence of bout duration on agreement between accelerometry data and self-reported physical activity. Med Sci Sports Exerc. 2006;38:S560.
34. Hamilton MT, Healy GN, Dunstan DW, Zderic TW, Owen $\mathrm{N}$. Too little exercise and too much sitting: inactivity physiology and the need for new reccomendations on sedentary behavior. Current Cardiovascular Risk Report. 2008;2:292-298.

35. Healy GN, Dunstan DW, Salmon J, Shaw JE, Zimmet PZ, Owen N. Television time and continuous metabolic risk in physically active adults. Med Sci Sports Exerc. 2008;40(4):639-645.

36. Katzmarzyk PT, Church TS, Craig CL, Bouchard C. Sitting Time and Mortality from All Causes, Cardiovascular Disease, and Cancer. Med Sci Sports Exerc. 2009:41:9981005.

37. Melanson E, Freedson P. Validity of the Computer Science and Applications, Inc. (CSA) activity monitor. Med Sci Sports Exerc. 1995;27:934-940.

38. Freedson PS, Melanson D, Sirard J. Calibration of the Computer Science and Applications (CSA), Inc. accelerometer. Med Sci Sports Exerc. 1998;30(5):777-781.

39. Swartz AM, Strath SJ, Bassett DR, Jr, O'Brien AM, King GA, Ainsworth BE. Estimation of energy expenditure using CSA accelerometers at hip and wrist sites. Med Sci Sports Exerc. 2000;32(9, Suppl):S450-S456.

40. Centers for Disease Control and Prevention (CDC). Adult participation in recommended levels of physical activityUnited States, 2001 and 2003. MMWR Morb Mortal Wkly Rep. 2005;54:1208-1211. 